\title{
Vitamin-D3-Überdosierungen bei Kleinkindern
}

\author{
Die chronischen Intoxikationsfälle mit Vitamin D3 haben zugenommen. Ein Grund \\ hierfür ist vermutlich die neue Formulierung mit einer höheren Konzentration. Beim \\ Produkt auf öliger Basis muss nur noch ein Tropfen statt vier Tropfen pro Tag verab- \\ reicht werden.
}

Christine Rauber-Lüthy, Cornelia Reichert, Hugo Kupferschmidt

Es bestehen keine Interessensbindungen.

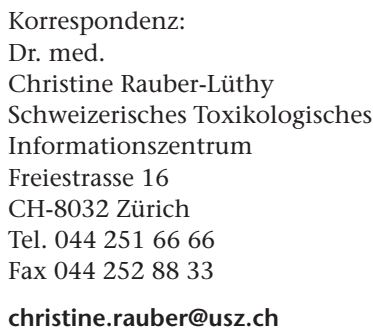

Vitamin D3 wird Kleinkindern schon seit den 40er Jahren als Rachitisprophylaxe verabreicht, anfänglich 300000-600000 IE als Stosstherapie alle 3 Monate, seit den 60er Jahren wird Vitamin D3 täglich in einer Dosis von 400 IE verabreicht. In den letzten Jahren wurden von Eltern und einem Teil des Medizinalpersonals Bedenken geäussert, weil das eingesetzte Präparat Alkohol als Lösungsmittel enthielt. Dies führte dazu, dass ölige Tropfen im Ausland gekauft oder von Apotheken selbst hergestellt wurden. Seit Anfang 2010 ist nun ein Präparat auf der Basis einer öligen Lösung in der Schweiz registriert.

\section{Akute Überdosierungen}

Überdosierungen mit Vitamin D3 bei Kleinkindern werden beim Schweizerischen Toxikologischen Informationszentrum (STIZ) seit Jahren regelmässig registriert. Dazu kommt es typischerweise, wenn die Fläschchen auf dem Wickeltisch oder anderswo im Kinderzimmer so platziert werden, dass 1- bis 4-Jährige sie ungehindert erreichen können. Solche akuten Ereignisse verlaufen in der Regel unproblematisch. Die Eltern oder Betreuer können vom STIZ beruhigt werden, weil selbst die einmalige Einnahme des Inhaltes eines ganzen Fläschchens mit 45000-200000 IE keine Intoxikationsgefahr birgt.

\section{Chronische Überdosierungen}

In den letzten drei Jahren registrierte das STIZ eine Zunahme der chronischen Intoxikationsfälle mit Vitamin D3 (Abb. 1). Dafür gibt es mehrere mögliche Erklärungen: Eine Rolle spielt sicher die neue Formulierung mit einer höheren Konzentration. Beim Produkt auf öliger Basis müssen nicht mehr 4 Tropfen pro Tag wie bei der alkoholischen Lösung, sondern es muss nur noch ein einziger Tropfen täglich verabreicht werden. Eltern, die von früher die Verabreichung von 4 Tropfen gewohnt sind, sind sich dieser Änderung zum Teil nicht bewusst. Eine weitere Fehlerquelle besteht darin, dass die Produkte mit öliger Lösung aus einer Pipette, das alkoholische Präparat aber direkt aus der Flasche getropft wird; dies suggeriert fälschlicherweise, dass eine ganze Pipette $(1 \mathrm{~mL})$ verabreicht werden soll. Wird somit statt eines Tropfens täglich eine ganze Pipette verabreicht, kommt es rasch zu toxischen Vitamin-D-Spiegeln.

In der Literatur lassen sich keine genauen Angaben zu toxischen Dosen bei chronischer Einnahme finden. Als maximale tägliche Einnahmemenge werden bei Kindern von 0-10 Jahren 1000 IE/d angegeben [1]. Diese Menge wird auch bei Einnahme über lange Zeit als sicher eingestuft. Es wird davon ausgegangen, dass 40000 IE täglich über 1-4 Monate bei Kleinkindern

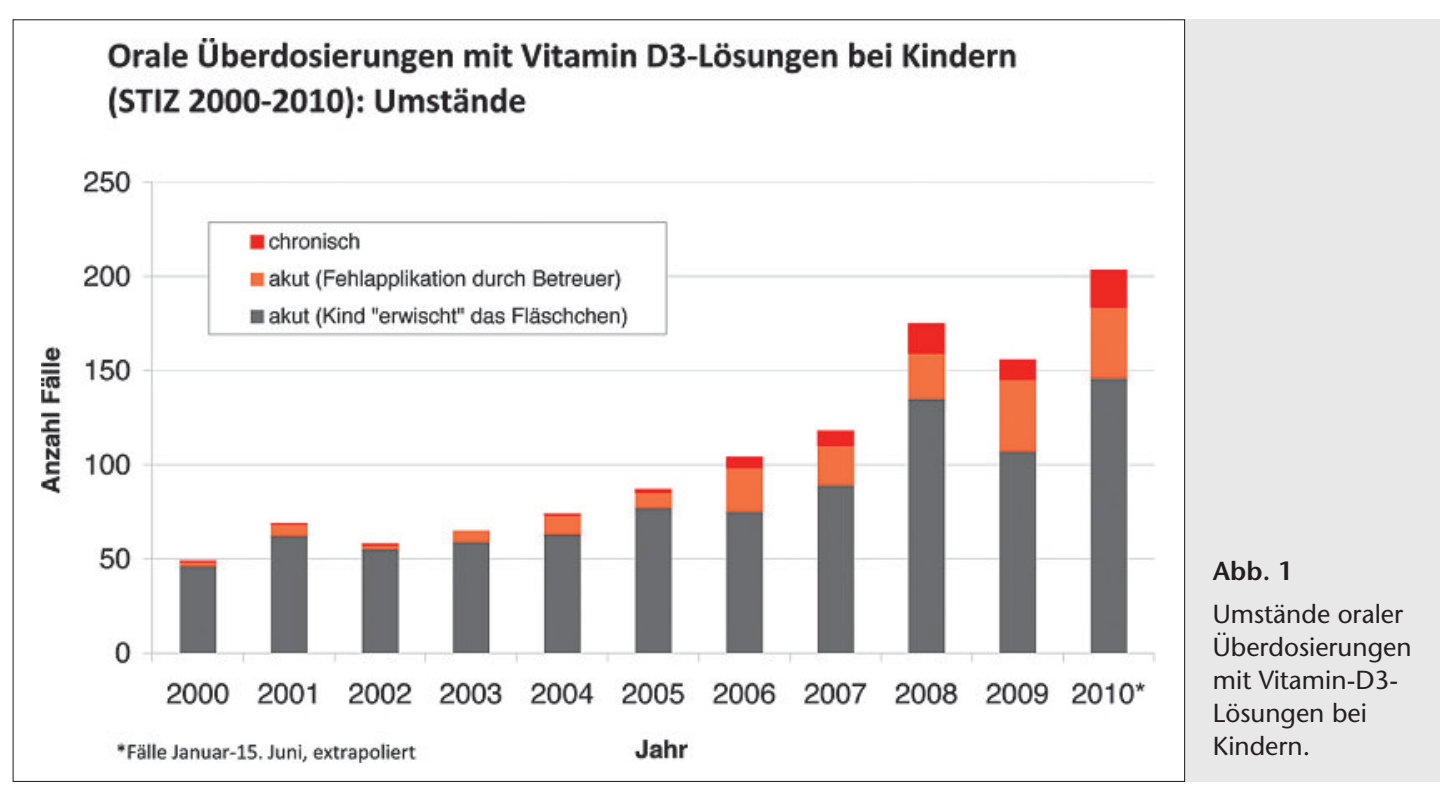


zu Toxizität führen können [2]. Bei Überdosierungen über Jahre hinweg können schon 2000-4000 IE täglich eine Intoxikation verursachen [2, 3].

In der Literatur werden einige Fälle mit schwerem Verlauf und bekannter verabreichter Dosis beschrieben [4-9]. Die Dosis von 600000 IE Vitamin D3 führte bei einem 4-monatigen Mädchen [6] und bei einem 35-tägigen Knaben [9] zu einer Nephrokalzinose. Weitere schwere Vergiftungen wurden nach chronischer Verabreichung von insgesamt 0,9 bzw. 1,2 Mio. IE beobachtet [6, 7]. Bei diesen Kindern wurde Nausea, Erbrechen, Obstipation, Anorexie, Lethargie, Polyurie, muskuläre Hypotonie, und je einmal eine Polydypsie sowie eine arterielle Hypertonie beschrieben. Es fanden sich Serumcalciumspiegel von 3,7 bis $4,6 \mathrm{mmol} / \mathrm{l}$ (normal $<2,7 \mathrm{mmol} / \mathrm{l}$ ) und Vitamin-D-Spiegel von 106 bis $340 \mathrm{ng} / \mathrm{ml}$ (normal 10-68 ng/ml). Die Hypercalcämie wurde mit Furosemid, Calcitonin, Biphosphonaten und Corticosteroiden behandelt.

\section{Eltern müssen ausdrücklich darauf hingewiesen werden, dass von der öligen Lösung täglich nur 1 Tropfen gegeben werden darf}

Am Schweizerischen Toxikologischen Informationszentrum wurden im letzten Jahr zwei schwere Fälle mit Nephrokalzinose registriert. Beide wurden durch hochkonzentrierte Lösungen verursacht. Ein 1-monatiger Knabe erhielt vier Wochen lang täglich 40000-60000 IE (total ca. 1400000 IE), und ein 1-monatiges Mädchen über 12 Tage insgesamt 2700000 IE Vitamin D3. In der Kasuistik des STIZ finden sich im Jahr 2009 elf weitere Fälle und in den ersten 5,5 Monaten des Jahres 2010 neun Fälle mit chronischer Überdosierung. Bei diesen zwanzig Fällen wurde die Fehldosierung aber so früh bemerkt, dass keine oder nur leichte Intoxikationssymptome auftraten.

\section{Prophylaxe}

Die verschreibenden Pädiater und die Apotheken spielen bei der Verhinderung von chronischen Intoxikationen mit Vitamin D3 eine zentrale Rolle. Schon im
Rahmen der Abgabe müssen die Eltern ausdrücklich darauf aufmerksam gemacht werden, dass von der öligen Lösung täglich nur 1 Tropfen gegeben werden darf. Schwere Vergiftungen können aber selbst dann noch verhindert werden, wenn eine Fehldosierung erst bei der Abgabe des zweiten Fläschchens bemerkt wird. Wenn Eltern oder Betreuer zu früh «Nachschub» holen, muss dies kritisch hinterfragt werden. Zudem sollten niemals die Ampullen mit 300000 IE zur parenteralen Therapie an die Patienten abgegeben werden.

\section{Literatur}

1 European Commission Health and Consumer Protection Directorate-General, Scientific Committee on Food. Opinion of the Scientific Committee on Food on the tolerable upper intake level of vitamin D (SCF/CS/NUT/UPPLEV/38 Final 16, December 2002). http://ec.europa.eu/food/fs/sc/scf/out157_en.pdf (Zugriff am 20. Juni 2010).

2 Dart RC (Hrsg.). Medical Toxicology. 3rd ed. Philadelphia: Lippincott Williams \& Wilkins; 2004

3 von Mühlendahl KE, Oberdisse U, Bunjes R, Brockstedt M. Vergiftungen im Kindesalter. 4. Aufl. Stuttgart: Thieme Verlag; 2003.

4 Gurkan F, Davutoglu M, Bosnak M, Ece A, Dikici B, Bilici M, Haspolat K. Pamidronate treatment in acute vitamin D intoxication. J Endocrinol Invest. 2004;27:680-2.

5 Barrueto F, Wang-Flores $\mathrm{HH}$, Howland MA, Hoffman RS, Nelson LS. Acute vitamin D intoxication in a child. Pediatrics. 2005;116:453-6.

6 Hatun S, Cizmecioğlu F. Use of alendronate in the treatment of vitamin D intoxication in infants. Turk J Pediatr. 2005;47:373-5.

7 Bereket A, Erdogan T. Oral bisphosphonate therapy for vitamin D intoxication of the infant. Pediatrics 2003;111:899-901.

8 Chambellan-Tison C. Horen B, Plat-Wilson G, Moulin P, Claudet I. Hypercalcémie majeure secondaire à une intoxication par la vitamine $\mathrm{D}$. Arch Pediat. 2007;14:1328-32.

9 Doneray H, Ozkan B, Caner I, Ozkan A, Karakelleoglu C. Intragastric alendronate therapy in two infants with vitamin D intoxication: A new method. Clin Toxicol. 2008;46:300-2. 